\title{
Image Spillover
}

National Cancer Institute

\section{Source}

National Cancer Institute. Image Spillover. NCI Thesaurus. Code C94913.

Phenomenon in a reconstructed image where true activity concentration of an object is not displayed totally in its corresponding pixels (voxels). Instead it is spatially smeared into nearby pixels (voxels) corresponding to its surrounding medium. 